\title{
Shaping and Forming Comes in All Shapes and Forms
}

\author{
DMITRY ESKIN (iD) ${ }^{1,2}$ \\ 1.-Brunel University London, Uxbridge, UK. 2.—e-mail: dmitry.eskin@brunel.ac.uk
}

The work of a $J O M$ subject editor is tricky at times. Several articles are submitted once the topic is announced, and it is the task of the editor to decide whether this or that article fits the topic even before it is sent to peer review.

In this case, it was challenging to interpret "shaping and forming" as, on the one hand, all metals are shaped and formed in various ways, whereas on the other hand, one needs to be selective in deciding what fits within the context of the current issue, along with being interesting and innovative.

The articles featured in this issue represent different technologies (hot gas pressure forming, extrusion, powder metallurgy, semisolid processing, and melt shearing), different type of materials (alloys, composites, and slurries), and a variety of research methods (processing maps, mechanical testing, bonding, thermodynamic and thermomechanical modeling, and physical simulation).

Although these articles are focused on specific materials and technologies, together they successfully highlight the breadth of the topic and represent the current state of the art.

The following articles are published under the topic "Aluminum: Shaping and Forming" in the April 2017 issue (vol. 69, no. 4) of JOM and can be accessed via the JOM page at http://link.springer. com/journal/11837/69/4/page/1:
- "Evaluation of Shearing Time Sufficient for Effective Liquid Metal Processing" by Agnieszka Dybalska, Dmitry Eskin, and Jayesh B. Patel.

- "Study on Hot Workability of Al-5.87Zn2.07Mg-2.28Cu Alloy Using Processing Map" by Dan Xiao, Xiaoyan Peng, Xiaopeng Liang, Ying Deng, Guofu Xu, and Zhimin Yin.

- "Physical Simulation Method for the Investigation of Weld Seam Formation During the Extrusion of Aluminum Alloys" by Gang Fang, Duc-Thien Nguyen, and Jie Zhou.

- "Integral Hot Gas Pressure Forming of an AA2219 Aluminum Ellipsoidal Shell" by S.J. Yuan, R. Zhang, and W.W. Zhang.

- "Alloying Behavior and Properties of AlBased Composites Reinforced with $\mathrm{Al}_{85} \mathrm{Fe}_{15}$ Metallic Glass Particles Fabricated by Mechanical Alloying and Hot Pressing Consolidation" by Lanxiang Zhang, LiKun Yang, Jinfeng Leng, Tongyang Wang, and Yan Wang.

- "Microstructures and Properties of TiCoated $\mathrm{SiC}_{\mathrm{p}}$ Reinforced Al-Si Alloy Composites" by Yan Feng, Junpeng Ren, Cuige Dong, and Richu Wang.

- "Effect of Temperature on Deformation Behavior of Sintered Porous AA2024 During Semisolid Compression" by Min Wu, Yunzhong Liu, Zhaoyubo Zeng, and Wenyan Luo. 